\title{
Pertussis Impfschutz ist wichtig!
}

Sanofi Pasteur informiert über neues Impfschema bei den 6-fach-Impfstoffen und über Schwangeren-Impfung gegen Pertussis.

Anlässlich des Weltkindertages 2020 hat Sanofi Pasteur mit einer WebPressekonferenz auf den wichtigen Pertussis-Impfschutz von Säuglingen, Kleinkindern und auch Erwachsenen aufmerksam gemacht. Über das neue Impfschema bei der 6-fach-Immunisierung gegen Polio, Diphtherie, Tetanus, Pertussis, Hib und Hepatitis B sowie über die Pertussis-Impfung bei Schwangeren sprachen Dr. med. Burkhard Lawrenz, Facharzt für Kinder- und Jugendmedizin, und Neonatologe Dr. med. Michael Wojcinski, Facharzt für Frauenheilkunde und Geburtshilfe, in ihren Vorträgen.

\section{STIKO: Reduzierung des Impf- schemas auf 2+1 Dosen}

Bislang erhielten reifgeborene Säuglinge vier Dosen eines hexavalenten Impfstoffes zur Grundimmunisierung gegen Diphtherie, Tetanus, Pertussis, Poliomyelitis, Haemophilus influenzae Typ b und Hepatitis B. Im Juni

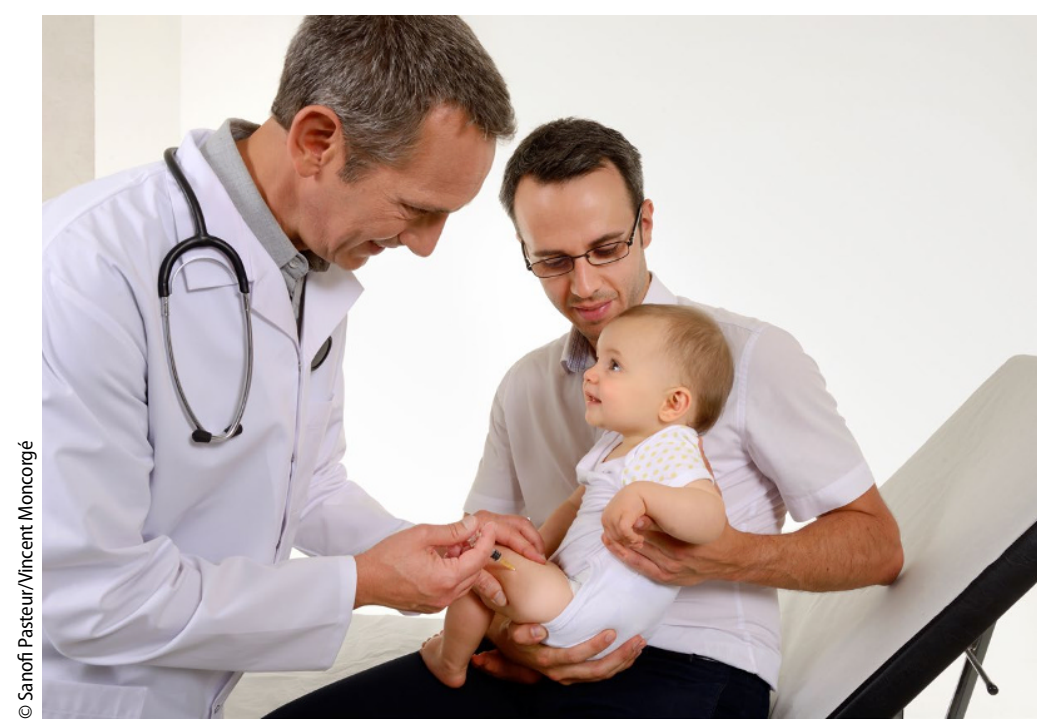

॥ Im Juni 2020 hat die ständige Impfkommission (STIKO) ihre Empfehlung für die Sechsfachimpfung aktualisiert und empfiehlt seitdem das reduzierte „2+1 Impfschema“.
2020 hat die ständige Impfkommission (STIKO) ihre Empfehlung für die Sechsfachimpfung aktualisiert und empfiehlt seitdem das reduzierte "2+ 1 Impfschema".

Das neue Impfschema wird bereits in vielen Ländern eingesetzt und als ausreichend wirksam gegen alle sechs Erkrankungen erachtet [1]. Es soll den Impfplan vereinfachen und Arzttermine für Säuglinge und Eltern einsparen. „Mit dem 2+1 Schema ist die zeitgerechte Impfung sehr wichtig. Damit die Wirksamkeit nicht beeinträchtigt wird, müssen alle drei Impfungen möglichst genau im von der STIKO empfohlenen Alter von 2, 4 und 11 Monaten erfolgen“, erläutert Dr. med Burkhard Lawrenz.

Reifgeborene Säuglinge sollen zukünftig mit drei Impfdosen im Alter von 2, 4 und 11 Monaten geimpft werden. Frühgeborene, die vor der vollendeten 37. Schwangerschaftswoche zur Welt kommen, erhalten

weiterhin vier Impfstoffdosen nach dem 3+1 Impfschema im Alter von 2, 3, 4 und 11 Monaten [1]. Der Kombinationsimpfstoff Hexyon ${ }^{\circledR}$ von Sanofi Pasteur wird bereits in vielen europäischen Ländern nach diesem Schema verimpft. Weltweit wurden bereits 107 Millionen Hexyon ${ }^{\circledast}$-Dosen ausgeliefert [2].

\section{Pertussis-Impfung bei Schwangeren}

2019 wurden beim Robert-Koch-Institut (RKI) 10.147 Pertussis-Fälle in Deutschland gemeldet [3]. „Pertussis ist keine reine Kinderkrankheit, sondern kann Menschen in jedem Alter betreffen. Neugeborene sind dadurch besonders gefährdet. Eine Impfung der Schwangeren gegen Pertussis kann die werdende Mutter und das Neugeborene mit einem Pieks schützen“, erläutert Dr. med. Michael Wojcinski. Mit einer Inzidenz von 51,6 Erkrankten/100.000 sind Säuglinge unter einem Jahr jedoch besonders gefährdet und haben mit $42,6 \%$ die höchste Hospitalisierungsrate [4].

Gerade in diesem Jahr ist es für Menschen jeden Alters wichtig, die ImpfEmpfehlungen des RKI zu befolgen und sich gegen impfpräventable Atemwegserkrankungen zu schützen, um eine gefährliche Doppelinfektion mit COVID-19 zu vermeiden [5].

Seit März 2020 empfiehlt die STIKO die Impfung gegen Pertussis für schwangere Frauen zu Beginn des dritten Trimenons bzw. bei erhöhter Wahrscheinlichkeit einer Frühgeburt im zweiten Trimenon. Diese Impfempfehlung während der Schwangerschaft zielt darauf ab, die Zahl der Pertussis-Infektionen, Krankenhausaufenthalte und Todesfälle bei Neugeborenen und Säuglingen zu verringern 


\section{Springer Medizin}

[6]. Covaxis ${ }^{\circledast}$ und Repevax ${ }^{\oplus}$ von Sanofi Pasteur sind die einzigen AuffrischImpfstoffe mit entsprechender Indikation [7]. Für junge Säuglinge kann Keuchhusten lebensbedrohlich sein. Da sie in den ersten Lebenswochen noch nicht geimpft werden können, ist eine Infektion mit Pertussis möglich. Eltern, Familienmitglieder und Angehörige im engen Umfeld sind die häufigste Infektionsquelle [8]. Säuglinge sind deshalb auf Antikörper angewiesen, die sie bereits vor der Geburt über die Plazenta der Mutter erhalten haben. Liegt die Impfung der Mutter jedoch längere Zeit zurück, kann der Keuchhusten-Schutz über die maternalen Antikörper zu schwach sein.

Die STIKO empfiehlt Auffrischungsimpfungen gegen Pertussis im Vorschul- und Jugendalter sowie einmalig bei der nächsten fälligen TetanusDiphtherie-Auffrischungsimpfung im Erwachsenenalter [6].

\section{Literatur}

1. RKI. Epid Bull $26 / 2020$

2. Fachinformation Hexyon ${ }^{\oplus}$, Stand Juni 2020

3. RKI. Epid Bull Nr. 03/2020, 17

4. RKI. Epid Bull 11. April 2019/15

5. Robert Koch-Institut zu Covid-19-Ausbruch „Wir können alle etwas tun, um das Virus einzudämmen." https://www.spiegel.de/ wissenschaft/medizin/coronavirus-wirkoennen-alle-etwas-tun-um-das-virusin-deutschland-einzudaemmen-a-2cfed4fc-c9f1-4200-85a3-00b06b2a9b1d.

Zugegriffen: 21. Aug. 2020

6. RKI. Epid Bull 13/2020

7. Fachinformation $\mathrm{COVAXIS}^{\circledast}$, Stand Februar 2019 und Fachinformation REPEVAX ${ }^{\circledast}$, Stand Februar 2019

8. Pertussis Vaccines: WHO position paper August 2015, https://www.who.int/wer/ 2015/wer9035.pdf?ua=1, Zugegriffen: 6. Aug. 2020
Hinweis des Verlags. Der Verlag bleibt in Hinblick auf geografische Zuordnungen und Gebietsbezeichnungen in veröffentlichten Karten und Institutsadressen neutral.

Paediatr. Paedolog. 2021 · 56:40-41 https://doi.org/10.1007/s00608-02100864-y

() Springer-Verlag GmbH Austria, ein Teil von Springer Nature 2021
Quelle: Pressemeldung Sanofi Pasteur, 21.09.2020 MAT-DE-2002212 (08/2020)

Hier steht eine Anzeige. 